\title{
Collision effects on the saturated electrostatic potential along a magnetic field line
}

\author{
I. Katanuma, ${ }^{\text {a) }}$ Y. Tatematsu, K. Ishii, T. Saito, and K. Yatsu \\ Plasma Research Center, University of Tsukuba, Tsukuba-City 305-8577, Japan
}

(Received 22 August 2002; accepted 13 December 2002)

\begin{abstract}
The Coulomb collision effects on a saturated electrostatic potential (plug potential) formation in the end-mirror cell of a tandem mirror were investigated by a Monte Carlo simulation of ion orbits. A non-Maxwellian electron distribution function, which leads to a modified Boltzmann law, is assumed to obtain the electrostatic potential. An ion velocity distribution is determined by the Monte Carlo simulation of ions. It was found that a saturated electrostatic potential is formed in a wide range of the Coulomb collisionality. Especially, fewer Coulomb collisions were found to create a higher saturated electrostatic potential along a magnetic field, although the Coulomb collisions are necessary for a saturated electrostatic potential formation. (C) 2003 American Institute of Physics.
\end{abstract} [DOI: $10.1063 / 1.1544536]$

\section{INTRODUCTION}

In the area of nuclear fusion, it has been proposed that the electrostatic potential is created along a magnetic field line positively to improve the confinement of plasmas along magnetic field lines in an open-ended magnetic confinement device, ${ }^{1-5}$ which is called a "tandem mirror." The subsequent experimental efforts at demonstrating the electrostatic potential formation along a magnetic field line have revealed that the electrostatic potential in the end-mirror cells in a tandem mirror is created by a different mechanism from the theoretical concept. ${ }^{6,7}$ That is, a high energy, magnetically trapped ion population is not necessary for electrostatic potential formation along a magnetic field line, and the electrons do not obey the traditional Boltzmann law in the entire region of the end-mirror cells of a tandem mirror.

Recently, a basic experiment has been carried out to demonstrate the electrostatic potential formation in a magnetic mirror when electron cyclotron resonance heating (ECRH) is applied to heat electrons in a magnetic mirror, ${ }^{8}$ where the experimental condition is similar to the present tandem mirror except that the ratio of Debye length to the system size is very different from each other. An electrostatic particle simulation was performed with the same condition as the above basic experiment and illustrated electrostatic potential formation in a mirror cell. ${ }^{9}$ The essential mechanism of electrostatic potential formation is that the charge separation of ions and electrons occurs due to the suppression of electron motion along a magnetic field line by ECRH.

On the other hand, we have carried out a Monte Carlo simulation ${ }^{10-12}$ by taking into account Coulomb collisions and have shown that the electrostatic potential can be formed along a magnetic field line in the mirror cell, ${ }^{13,14}$ where the

${ }^{a)}$ Electronic mail: katanuma@prc.tsukuba.ac.jp condition used in the Monte Carlo simulation is similar to that of a tandem mirror experiment. The major differences of our work ${ }^{13,14}$ from the previous work ${ }^{8,9}$ is that, first, the effects of Coulomb collisions are included in the dynamics of ions along a magnetic field line and, second, the charge neutrality condition is used to obtain the electrostatic potential, so that the electrons do not obey the traditional Boltzmann law in our electrostatic potential mechanism. Therefore, the field aligned electrostatic potential formation ${ }^{13,14}$ corresponds to a presheath potential formation mechanism, ${ }^{15}$ while the electrostatic potential formation ${ }^{8,9}$ is a sheath potential formation.

The Coulomb collisions are plausible candidates for a saturated electrostatic potential formation along a magnetic field line in a mirror cell because the plasma production required to maintain the charge neutral condition for a saturated electrostatic potential formation (a plug potential formation) is larger than the amount of present tandem mirror experiments. ${ }^{16}$ A saturated electrostatic potential formation has been demonstrated by a Monte Carlo simulation in Ref. 14 , where the ratio of Coulomb collision time to ion transit time with thermal speed from $z_{b}$ to $z_{m}$ was fixed. The purpose of this article, therefore, is to make clear the dependence of a magnitude of Coulomb collisions required for the saturated electrostatic potential formation on the resultant maximum of electrostatic potential (plug potential).

\section{MODIFIED BOLTZMANN LAW}

Henceforth we consider a neutral plasma. Due to the smaller mass of electrons than that of ions, electrons are assumed to satisfy the pressure balance along a magnetic field line, i.e., inertia terms are neglected in the equation of motion. If the electrons have an isotropic pressure with constant temperature $T_{e}$, the electron density $n_{e}$ obeys the traditional Boltzmann law, that is, $e(\varphi(z)-\varphi(0))$ $=T_{e} \ln \left\{n_{e}(z) / n_{e}(0)\right\}$, where $e$ is unit charge and $\varphi(z)$ is the magnitude of electrostatic potential at the axial coordinate $z$. 
The electron distribution function usually deviates from Maxwellian greatly when electrons are heated by strong ECRH. ${ }^{17}$ In the end-mirror cell (plug/thermal barrier region) of a tandem mirror, electrons are heated by a strong ECRH to create magnetically trapped high energy electrons. The nonMaxwellian electrons no longer obey the traditional Boltzmann law. To see this we assume the electron distribution function in the region $z_{b} \leqslant z \leqslant z_{m}$, shown in Fig. 1 as

$$
f_{e}=\left\{\begin{array}{l}
n_{e c}\left(\frac{m_{e}}{2 \pi T_{e c}}\right)^{3 / 2} \exp \left\{-\frac{\varepsilon+e \varphi_{i}}{T_{e c}}\right\} \quad \text { for } \varepsilon \geqslant \mu B_{i}-e \varphi_{i}, \\
n_{e c}\left(\frac{m_{e}}{2 \pi T_{e c}}\right)^{3 / 2} \exp \left\{-\frac{\varepsilon+e \varphi_{i}-\alpha_{e} \mu B_{i}}{\left(1-\alpha_{e}\right) T_{e c}}\right\} \quad \text { for } \varepsilon<\mu B_{i}-e \varphi_{i},
\end{array}\right.
$$

where $T_{e c}$ and $n_{e c}$, the electron temperature and density at $z=z_{i}, m_{e}$, electron mass, and $B$ is the magnetic field strength. The quantities $\varepsilon$ and $\mu$ are the electron total energy $\left(\varepsilon=\frac{1}{2} m_{e} v^{2}-e \varphi\right)$ and magnetic moment $\left(\mu=\frac{1}{2} m_{e} v_{\perp}^{2} / B\right)$, where $v$ is velocity and $v_{\perp}$ is the velocity component perpendicular to a magnetic field. A constant $\alpha_{e}$ gives an anisotropy of electron distribution function, but the $\alpha_{e}$ should satisfy the condition $\alpha_{e} \leqslant B_{b} / B_{i}$ in order that the electron distribution function (1) does not have to be infinite in the region $B_{b} \leqslant B \leqslant B_{m}$ (i.e., $z_{b} \leqslant z \leqslant z_{m}$ ) when $\mu \rightarrow \infty$ with $\varepsilon$ $=\mu B$. The subscripts $i$ and $m$ denote the quantity at $z=z_{i}$ and at $z=z_{m}$, and, henceforth, we assume $B_{i}=B_{m}$ for simplicity.

In the definition of the electron distribution function (1) the electrons, which are Maxwellian at $z=z_{i}$, flow into the mirror cell $\left(z_{i} \leqslant z \leqslant z_{m}\right)$ and the electrons trapped in the mirror cell are heated by ECRH to be non-Maxwellian. Here the electrons are assumed to be supplied continuously at $z=z_{i}$ with a Maxwellian distribution function.

The electron density $n_{e}(z)\left(z_{b} \leqslant z \leqslant z_{m}\right)$ is obtained by integrating Eq. (1) in the appropriate velocity space as

$$
\begin{aligned}
n_{e}(z)= & \frac{B(z) n_{e c}}{\sqrt{\pi} T_{e c}^{3 / 2}}\left(\left[\int_{\mu B_{b}-e \varphi_{b}}^{\infty} \mathrm{d} \varepsilon \int_{0}^{\mu_{e * *}} \mathrm{~d} \mu\right.\right. \\
& \left.+\int_{\mu B_{i}-e \varphi_{i}}^{\infty} \mathrm{d} \varepsilon \int_{\mu_{e * *}}^{\infty} \mathrm{d} \mu\right] \\
& \times \frac{1}{(\varepsilon-\mu B(z)+e \varphi(z))^{1 / 2}} \exp \left\{-\frac{\varepsilon+e \varphi_{i}}{T_{e c}}\right\} \\
& +\int_{\mu B(z)-e \varphi(z)}^{\mu B_{i}-e \varphi_{i}} \int_{\mu_{e *}}^{\infty} \mathrm{d} \mu \frac{(\varepsilon-\mu B(z)+e \varphi(z))^{1 / 2}}{(\varepsilon)} \\
& \left.\times \exp \left\{-\frac{\varepsilon+e \varphi_{i}-\alpha_{e} \mu B_{i}}{\left(1-\alpha_{e}\right) T_{e c}}\right\}\right) .
\end{aligned}
$$

Here $\mu_{e *} \equiv e\left(\varphi(z)-\varphi_{i}\right) /\left(B(z)-B_{i}\right)$ and $\mu_{e * *} \equiv e\left(\varphi_{b}\right.$ $\left.-\varphi_{i}\right) /\left(B_{b}-B_{i}\right)$. With the assumptions of $e\left(\varphi-\varphi_{b}\right) / T_{e c}$ $\gg 1$ and $\mu_{e * *} B_{i} / T_{e c} \gg 1$, the integration in Eq. (2) is carried out to be $\mathrm{b}^{14}$

$$
e\left(\varphi(z)-\varphi_{b}\right) \simeq\left(1-\alpha_{e}\right) T_{e c} \ln \left\{\frac{B_{b}\left[B(z)-\alpha_{e} B_{i}\right]}{B(z)\left[B_{b}-\alpha_{e} B_{i}\right]} \frac{n_{e}(z)}{n_{e b}}\right\}
$$

Here $n_{e b} \equiv n_{e}\left(z_{b}\right)$.

The distribution function of electrons trapped in the mirror cell in Eq. (1) is written as

$$
\begin{aligned}
f_{e}= & n_{e c}\left(\frac{m_{e}}{2 \pi T_{e c}}\right)^{3 / 2} \exp \left\{-\frac{\frac{1}{2} m_{e} v_{\|}^{2}}{\left(1-\alpha_{e}\right) T_{e c}}\right. \\
& \left.-\frac{\frac{1}{2} m_{e} v_{\perp}^{2}}{\left(1-\alpha_{e}\right)}+\frac{e\left(\varphi_{b}-\varphi_{i}\right)}{\left(1-\alpha_{e}\right) T_{e c}}\right\} \text { at } z=z_{b} .
\end{aligned}
$$

This electron distribution function $f_{e}$ is bi-Maxwellian with two component temperatures $T_{e \|}, T_{e \perp}$ parallel and perpendicular to the magnetic field line at $z=z_{b}$. Here $T_{e \|}=(1$ $\left.-\alpha_{e}\right) T_{e c}$ and $T_{e \perp}=\left(1-\alpha_{e}\right) /\left(1-\alpha_{e} B_{i} / B_{b}\right) T_{e c}$. Equation (3) reduces to

$$
e\left(\varphi(z)-\varphi_{b}\right)=T_{e \|} \ln \left\{\left[\frac{T_{e \perp}}{T_{e \|}}+\left(1-\frac{T_{e \perp}}{T_{e \|}}\right) \frac{B_{b}}{B(z)}\right] \frac{n_{e}(z)}{n_{e b}}\right\},
$$

by using $T_{e \|}$ and $T_{e \perp}$.

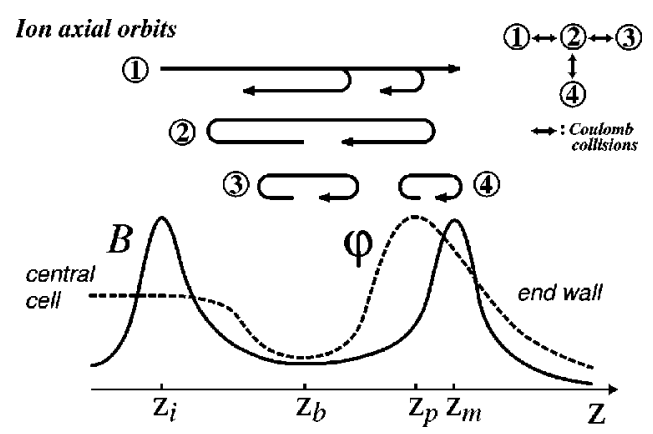

FIG. 1. Schematic diagram of the end mirror cell in a tandem mirror. An arrow $\rightarrow$ with a mark (1) represents the orbits of ions input from $z=z_{i}$. The arrows $\rightarrow$ with each mark (2), (3) and (4) are the orbits of ions trapped magnetically and electrostatically. The short arrow $\leftrightarrow$ represents the transition of ion orbit due to the Coulomb collisions. The solid curve shows a magnetic field, and dashed curve is an electrostatic potential. A local maximum of the electrostatic potential profile is assumed at $z=z_{p}$, which is called "plug point." 
Equation (4) is the modified Boltzmann law, which gives the relation of the electrostatic potential $\varphi(z)$ to the electron density $n_{e}(z)$. If the ion density is determined by solving the kinetic equation, the electrostatic potential is obtained by Eq. (4) and the charge neutrality condition $n_{e}(z)=n_{i}(z)$.

\section{RESULTS OF MONTE CARLO SIMULATION}

Ions have a larger inertia term than electrons in their kinetic equation so that ion kinetic effects should be taken into account for the electrostatic potential formation. It is known that the plasma presheath formation requires some mobility limited motion for ions ${ }^{15}$ or plasma production ${ }^{18}$ in order to maintain the charge neutrality condition. The saturated electrostatic potential formation in a mirror cell with non-Maxwellian electrons also requires such plasma production ${ }^{16,19}$ or the effects of Coulomb collisions suppressing the motion for ions. ${ }^{14}$

In order to take into account the Coulomb collisions on ions, we carry out a Monte Carlo simulation (the details of the code were described in Refs. 10-14). A brief description of the code is mentioned in the following. Let us consider the test ions with distribution function $f_{\text {test }}(\boldsymbol{v})=\delta(\boldsymbol{v}-\boldsymbol{U})$, where $\delta()$ is Dirac delta function and the test ions are assumed to have the same velocity $\boldsymbol{U}$ at time $t$. With the help of the linearized Fokker-Planck equation, ${ }^{10}$ the time evolutions of the test ion mean velocity moments reduce to

$$
\begin{aligned}
\frac{\partial \boldsymbol{U}}{\partial t} \equiv & \frac{\boldsymbol{U}}{U} \int \mathrm{d} \boldsymbol{v} \frac{\boldsymbol{U} \cdot \boldsymbol{v}}{U} \frac{\partial f_{\text {test }}(\boldsymbol{v})}{\partial t} \\
= & \boldsymbol{U} \sum_{\alpha=i, e}\left(1+\frac{m_{i}}{m_{\alpha}}\right)\left(2 \sqrt{\frac{a_{\alpha}}{\pi}} \frac{1}{U^{2}} \exp \left\{-a_{\alpha} U^{2}\right\}\right. \\
& \left.-\frac{1}{U^{3}} \operatorname{erf}\left\{\sqrt{a_{\alpha}} U\right\}\right) \Gamma_{\alpha}, \\
\frac{\partial U_{\perp}^{2}}{\partial t} \equiv & \int \mathrm{d} \boldsymbol{v}\left(\frac{\boldsymbol{U} \times \boldsymbol{v}}{U}\right)^{2} \frac{\partial f_{\text {test }}(\boldsymbol{v})}{\partial t} \\
= & \frac{2}{U} \sum_{\alpha=i, e}\left[\frac{1}{\sqrt{\pi a_{\alpha}}} \frac{1}{U} \exp \left\{-a_{\alpha} U^{2}\right\}\right. \\
& \left.+\left(1-\frac{1}{2 a_{\alpha} U^{2}}\right) \operatorname{erf}\left\{\sqrt{a_{\alpha}} U\right\}\right] \Gamma_{\alpha}, \\
\frac{\partial \Delta U_{\|}^{2}}{\partial t} \equiv & \int \mathrm{d} \boldsymbol{v}\left(\frac{\boldsymbol{U} \cdot(\boldsymbol{v}-\boldsymbol{U})}{U}\right)^{2} \frac{\partial f_{\text {test }}(\boldsymbol{v})}{\partial t} \\
= & \frac{2}{U} \sum_{\alpha=i, e}\left(-\frac{1}{\sqrt{\pi a_{\alpha}}} \frac{1}{U} \exp \left\{-a_{\alpha} U^{2}\right\}\right. \\
& \left.+\frac{1}{2 a_{\alpha} U^{2}} \operatorname{erf}\left\{\sqrt{a_{\alpha}} U\right\}\right) \Gamma_{\alpha},
\end{aligned}
$$

where $\Gamma_{\alpha} \equiv\left(4 \pi n_{\alpha} e^{4} / m_{i}^{2}\right) \ln \Lambda_{\alpha i}, a_{\alpha} \equiv m_{\alpha} / 2 T_{\alpha}$, and $\operatorname{erf}()$ is the error function. The test ions with velocity $\boldsymbol{U}$ at time $t$ have an average velocity $\boldsymbol{U}-\Delta \boldsymbol{U}$ after a time interval $\delta t$, where $\Delta \boldsymbol{U}=\delta t(\partial \boldsymbol{U} / \partial t)$. The probability of the ion having a velocity $\boldsymbol{U}-\Delta \boldsymbol{U}+\boldsymbol{v}_{\text {para }}+\boldsymbol{v}_{\text {perp }}$ at time $t+\delta t$ is given in terms of the probabilities $g_{\|}\left(\boldsymbol{v}_{\text {para }}\right)=\left(1 / \sqrt{2 \pi} \sigma_{\|}\right)$ $\times \exp \left\{-\left(\boldsymbol{v}_{\text {para }}-\Delta \boldsymbol{U}\right)^{2} / 2 \sigma_{\|}^{2}\right\}$, and $g_{\perp}\left(\boldsymbol{v}_{\text {perp }}\right)=\left(1 / \sqrt{2 \pi} \sigma_{\perp}\right)$ $\times \exp \left\{-\boldsymbol{v}_{\text {perp }}^{2} / 2 \sigma_{\perp}^{2}\right\}, \quad$ where $\quad \sigma_{\|}^{2}=\delta t\left(\partial \Delta U_{\|}^{2} / \partial t\right), \quad \sigma_{\perp}^{2}$ $=\delta t\left(\partial U_{\perp}^{2} / \partial t\right)$, and $\boldsymbol{v}_{\text {para }} \equiv \boldsymbol{v} \cdot \boldsymbol{U} / U, \boldsymbol{v}_{\text {perp }} \equiv \boldsymbol{v}-\boldsymbol{v}_{\text {para }}$. Here $g_{\|}\left(\boldsymbol{v}_{\text {para }}\right)$ and $g_{\perp}\left(\boldsymbol{v}_{\text {perp }}\right)$ give the probabilities of the test ion having the components of $\boldsymbol{v}_{\text {para }}$ and $\boldsymbol{v}_{\text {perp }}$. Further information on the procedure needed to give the effect of Coulomb collisions on the test ions will be found in Ref. 10.

We are interested in the electrostatic potential along a magnetic field line, so that ion orbits are calculated only along a magnetic field line, i.e.,

$$
\frac{\mathrm{d} z}{\mathrm{~d} t}=v_{\|}, \quad v_{\|}=\sqrt{\frac{m_{i}}{2}(\varepsilon-\mu B(z)-e \varphi(z))},
$$

where the $z$-coordinate is the axial coordinate, and magnetic field lines near the axis in a mirror device can be considered to be parallel to the axial coordinate within a long thin approximation (i.e., with the assumption of radial scale length $\ll$ axial scale length). Therefore, the coordinate along a magnetic field line coincides with the $z$-axis. The quantities $\varepsilon$ and $\mu$ are the ion total energy and magnetic moment, respectively.

Figure 1 illustrates the axial profile of a magnetic field under consideration and an expected steady state electrostatic potential profile. The ions are continuously supplied at $z_{i}$ with a Maxwellian distribution function of temperature $T_{i}$, the orbit of passing ions are illustrated by a symbol (1). The passing ions change to shallow trapped ions due to Coulomb collisions with field ions and electrons, and the shallow trapped ions denoted by a symbol (2) can pass through the electrostatic potential maximum (called plug) at $z_{p}$ in Fig. 1. Further Coulomb collisions make a shallow trapped ion into a deeply trapped ion, denoted by symbols (3) and (4) in Fig. 1 , and the deeply trapped ions cannot pass through the plug due to their low kinetic energy parallel to the magnetic field line. The deeply trapped ions accumulate in the mirror cell and, finally, the ions there become Maxwellian with the same temperature $T_{i}$ as that of the passing ions at $z_{i}$.

In order to take into account the effect of nonMaxwellian ions in the steady state, we include a radial loss to test ions artificially, as follows. That is, an ion loss time $\tau_{\text {loss }}$ is introduced. The uniform random number $\xi_{k}$ (a number from 0 to 1 ) is introduced for the $k$ th test ion. The number $\xi_{k}$ is compared with the magnitude of $\exp \left\{-t_{k} / \tau_{\text {loss }}\right\}$, where the time $t_{k}$ is measured from the time when the $k$ th test ion was input at $z_{i}$ in Fig. 1. If $\xi_{k}>\exp \left\{-t_{k} / \tau_{\text {loss }}\right\}$, the $k$ th test ion is lost from the mirror cell. Because we are looking for the steady state of electrostatic potential, the ions which were lost are input at $z_{i}$ immediately. Here the velocity components $v_{\|}$and $v_{\perp}$ of ion at $z_{i}$ are given to be Maxwellian with temperature $T_{i}$ in the passing region in the velocity space by means of a random number.

On the assumption of the charge neutrality condition, the electron density is the same as the ion density, i.e., $n_{e}(z)$ $=n_{i}(z)$. The electrostatic potential $\varphi(z)$ is determined by the modified Boltzmann law given by Eq. (4). In the simulation, the electrostatic potential is given in advance, i.e., 
$\varphi(z)=0$. The test ions are distributed uniformly along the axis (i.e., along the magnetic field line axis) initially. Here the mean temperature of initial test ions is $T_{i}$ at each axial position, and each velocity component of test ions is given by random numbers. The motion of ions is followed in the given electrostatic potential and the density is calculated by each ion position. ${ }^{14}$ The calculation of ion motions continues until the steady state of ion density is realized.

The new electrostatic potential is calculated by the modified Boltzmann law Eq. (4), with the ion density in the steady state in the old electrostatic potential profile. The ion motion is traced again in the new electrostatic potential profile and the ion density is accumulated after initialization until the steady state of ion density is realized. The above procedure is repeated until the steady state of both ion density and electrostatic potential profiles are realized.

The parameters used in the simulation are as follows. The magnetic field profile from $z=z_{b}$ to $z=z_{m}$ is adopted in the end mirror cell in the GAMMA10 tandem mirror, ${ }^{6}$ where the axial length $L_{z}$ from the thermal barrier $z=z_{b}$ to the outer mirror throat $z=z_{m}$ is $L_{z}=120 \mathrm{~cm}$. The temperatures $T_{\text {field }}$ of the field ions and electrons, with which the test ions receive the Coulomb collision, are $100 \mathrm{eV}$. The number density $n_{\text {field }}$, where the density of field ions is the same as that of field electrons, is $n_{\text {field }}=10^{11} \mathrm{~cm}^{-3}$, which is uniform along $z$. In this field plasma the deflection time $\tau_{D}$ is

$$
\tau_{D}=\sqrt{\frac{m_{i}}{2}} \frac{T_{\text {field }}^{3 / 2}}{\pi n_{\text {field }} e^{4} \ln \Lambda_{i i}} .
$$

Here $\ln \Lambda_{i i}$ is the Coulomb logarithm. The detailed procedure to include the effects of Coulomb collisions on test ions in the Monte Carlo code will be found in Refs. 10-12. The transit time $\tau_{\text {transit }}$ is defined as $\tau_{\text {transit }} \equiv L_{z} \times\left(T_{i} / m_{i}\right)^{-1 / 2}$, i.e., is the time necessary for a thermal test ion to move from $z$ $=z_{b}$ to $z=z_{m}$, where species of ions is hydrogen. The deflection time is $\tau_{D} \simeq 5.6 \times 10^{-3} \mathrm{~s}$ and the transit time is $\tau_{\text {transit }} \simeq 1.2 \times 10^{-5} \mathrm{~s}$ in the above parameters.

The temperature of test ions is set $T_{i}=100 \mathrm{eV}$ at $z$ $=z_{i}$. The test ions escaping from the outer mirror throat $z$ $=z_{m}$ or lost (radially) are input again immediately at the inner mirror throat $z=z_{i}$ in the simulation.

In order to save computer time only the region from $z$ $=z_{b}$ to $z=z_{m}$ is calculated. The test ions input at $z=z_{i}$ are mapped at $z=z_{b}$ with a positive velocity $v_{\|}$on the assumption of conservation of $\varepsilon$ and $\mu$ during its flight from $z=z_{i}$ to $z=z_{b}$. The test ions reached at $z=z_{b}$ with $v_{\|} \leqslant 0$ is reflected perfectly at $z=z_{b}$ if $\varepsilon \leqslant \mu B_{i}+e \varphi_{i}$, and is input again at $z$ $=z_{i}$ with Maxwellian velocity of $T_{i}$ if $\varepsilon>\mu B_{i}+e \varphi_{i}$.

The algorithm of ion supply to the end-mirror cell adopted in the Monte Carlo simulation is consistent with the present tandem mirror experiment, where the ions in the endmirror cell are supplied from the central cell and escape through the outer mirror throat or escape radially.

The electrostatic potential is set $\varphi_{i}=0$ at $z=z_{i}$. The potential $\varphi_{b}$ at $z=z_{b}$ is given in advance as a boundary condition of the electrostatic potential and is not changed through the simulation run. The potential profile $\varphi(z)$, therefore, is determined on the basis of its magnitude at $z=z_{b}$.
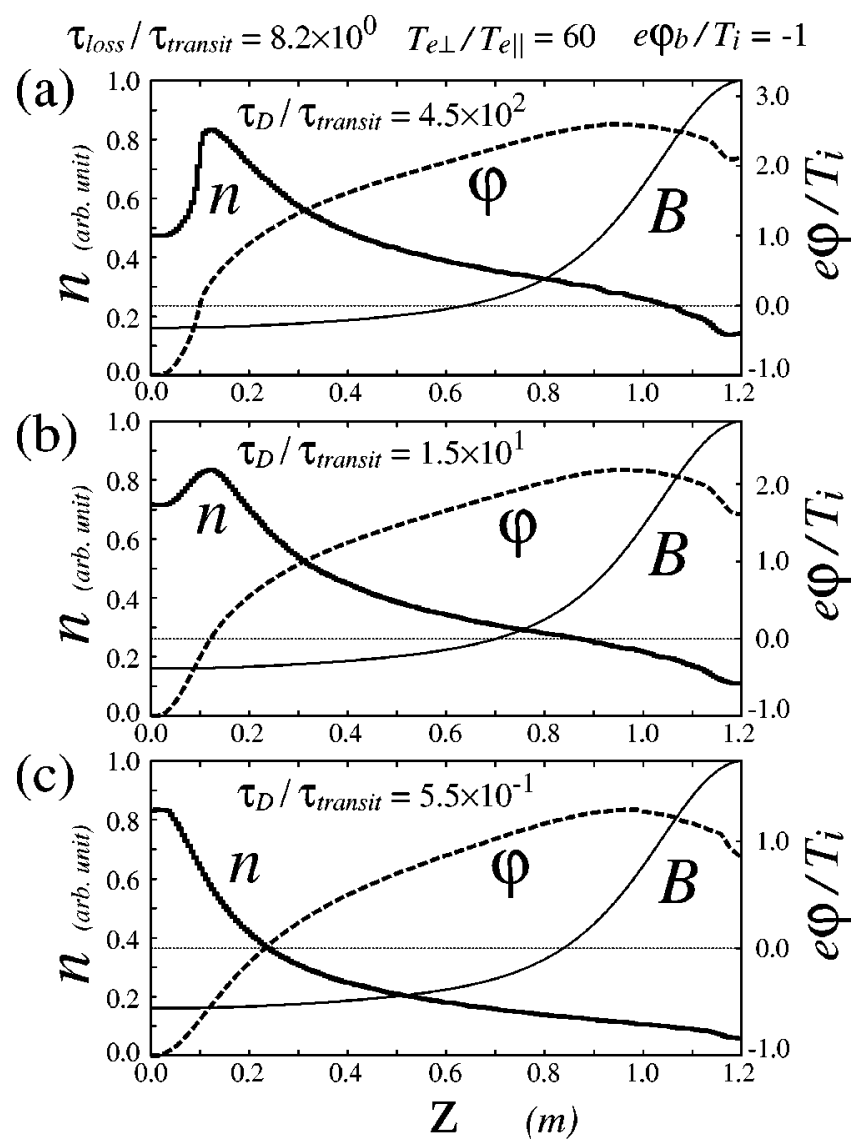

FIG. 2. Axial profiles of test ion density $n$ and electrostatic potential $\varphi$ along magnetic field $B$ in the steady state, which were obtained by Monte Carlo simulation. Here (a) is $\tau_{D} / \tau_{\text {transit }}=4.5 \times 10^{2}$, (b) is $\tau_{D} / \tau_{\text {transit }}=1.5$ $\times 10^{1}$, and (c) is the case of $\tau_{D} / \tau_{\text {transit }}=5.5 \times 10^{-1}$.

Figure 2 shows the steady state axial profiles of electrostatic potential and ion density obtained by the Monte Carlo simulations. Clearly, a maximum point (plug point) in the continuous saturated electrostatic potential profile is observed in Figs. 2(a), 2(b), and 2(c). As pointed out in previous work, ${ }^{19}$ the electrostatic potential can be discontinuous at the maximum point of the electrostatic potential without any dissipation, because the situation at the electrostatic potential maximum is the same as that at a sheath potential region. ${ }^{14,15,19}$ An effect of Coulomb collision in the Monte Carlo simulation prevents a sheath potential from forming at the maximum of an saturated axial electrostatic potential profile.

Figure 2(a) is the case of $\tau_{\text {transit }} \ll \tau_{\text {loss }} \ll \tau_{D}$, where ions trapped in a magnetic mirror cell are lost faster than those are supplied by collisional filling from passing ions. The result that the density around $z=0 \mathrm{~m}$ is lower than the density around $z=0.1 \mathrm{~m}$ in Fig. 2(a) comes from the reason why the ion collisional filling from passing region is slower than the loss from trapped region.

On the other hand, Fig. 2(c) is the case of $\tau_{D} \lesssim \tau_{\text {transit }}$ $\ll \tau_{\text {loss }}$, where ions lost from the magnetic mirror cell are supplied immediately by a collisional filling of passing ions. Therefore, the ion density is maximum at $z=0 \mathrm{~m}$ in Fig. 2(c).

Although the ion density axial profiles change greatly 


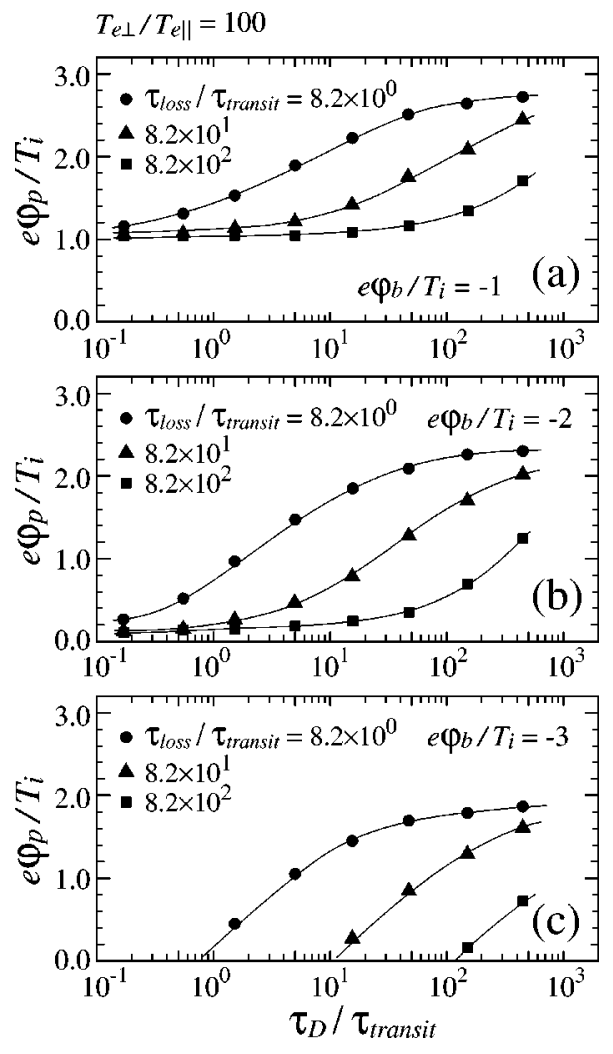

FIG. 3. The dependence of maximum electrostatic potential height $\varphi_{p}$ on the Coulomb collision time $\tau_{D}$ in the steady state obtained by Monte Carlo simulation. Here (a) is $e \varphi_{b} / T_{i}=-1$, (b) is $e \varphi_{b} / T_{i}=-2$, and (c) is the case of $e \varphi_{b} / T_{i}=-3$.

according to the parameters of $\tau_{D} / \tau_{\text {transit }}$ and $\tau_{\text {loss }} / \tau_{\text {transit }}$, the electrostatic potential axial profiles do not change as much, except for their magnitudes, in Figs. 2(a), 2(b), and 2(c). The passing ions have an average kinetic energy of $T_{i}+e \varphi_{b}$. The electrostatic potential is formed along a magnetic field line to retard the axial motion of ions, which overcome the electrons' electric force to maintain a charge neutrality condition in whole region of a magnetic mirror cell.

Figure 3 is the results of Monte Carlo simulation for various parameters of $\tau_{D}$ and $\tau_{\text {loss }}$. It is seen in Figs. 3(a) and 3(b) that the magnitudes of $e \varphi_{p} / T_{i}$ converge on the same value in the region of $\tau_{D} / \tau_{\text {transit }} \lesssim 1$ in each figure, where the effects of Coulomb collisions dominate the effects of ion loss on a time scale of $\tau_{\text {loss }}$. In the Coulomb collision the dominant regime over ion loss process, the ions accelerated by the electrostatic potential difference $\varphi_{b}$ from $z_{i}$ to $z_{b}$ are hidden into the majority of ions trapped in a mirror cell electrostatically, as well as magnetically. Therefore, the magnitude of $e\left(\varphi_{p}-\varphi_{b}\right) / T_{i}$ is the same in both Figs. 3(a) and 3 (b) in the region of $\tau_{D} / \tau_{\text {transit }} \lesssim 1$.

In the ion loss dominant regime over Coulomb collision, the majority of ions existing in the mirror cell is the passing ions accelerated by the electrostatic potential from $z_{i}$ to $z_{b}$. It is seen in Figs. 3(a), 3(b), and 3(c) that the magnitudes of $e \varphi_{p} / T_{i}$ with different $\tau_{\text {loss }} / \tau_{\text {transit }}$ converge on the same value even in the region of $\tau_{D} / \tau_{\text {transit }} \gg 1$, where the effects of Coulomb collisions can be neglected. This physical meaning is as follows. Coulomb collisions make the ion density $n_{i}(z)$ smooth around the potential maximum, i.e., $|\mathrm{d} \varphi(z) / \mathrm{d} z|<\infty$ at $z=z_{p},{ }^{14}$ in the case of which the magnitude of plug potential is determined by the charge neutrality condition of ions and electrons there. In the weak limit of collisions the ion density at $z=z_{p}$ is given by

$$
n_{i}\left(z_{p}\right)=\frac{1}{2} n_{e i} \exp \left\{-\frac{e\left(\varphi_{p}-\varphi_{i}\right)}{T_{i}}\right\}
$$

[see Eq. (14) in Ref. 14], where $n_{e i} \equiv n_{e}\left(z_{i}\right)$ and charge neutrality condition $n_{i}\left(z_{i}\right)=n_{e}\left(z_{i}\right)$ was used. On the other hand, the electron density $n_{e}\left(z_{p}\right)$ is given by

$$
n_{e}\left(z_{p}\right)=n_{e b}\left[\frac{T_{e \perp}}{T_{e \|}}+\left(1-\frac{T_{e \perp}}{T_{e \|}}\right) \frac{B_{b}}{B_{p}}\right]^{-1} \exp \left\{\frac{e\left(\varphi_{p}-\varphi_{b}\right)}{T_{e \|}}\right\}
$$

in Eq. (4). Therefore, the plug potential height $e\left(\varphi_{p}\right.$ $\left.-\varphi_{i}\right) / T_{i}$ approaches to

$$
\begin{aligned}
\frac{e\left(\varphi_{p}-\varphi_{i}\right)}{T_{i}}= & -\frac{e\left(\varphi_{i}-\varphi_{b}\right)}{T_{e \|}+T_{i}}+\left(\frac{T_{e \|}}{T_{e \|}+T_{i}}\right) \\
& \times \ln \left\{\left[\frac{T_{e \perp}}{T_{e \|}}+\left(1-\frac{T_{e \perp}}{T_{e \|}}\right) \frac{B_{b}}{B_{p}}\right] \frac{n_{e i}}{2 n_{e b}}\right\},
\end{aligned}
$$

in the weak limit of collisions, where Eq. (8) was obtained by the charge neutrality condition $n_{i}\left(z_{p}\right)=n_{e}\left(z_{p}\right)$ given above.

As mentioned previously in this article, the electrostatic potential from $z_{b}$ to $z_{m}$ is created by the ion kinetic energy. Therefore, the magnitude of $e\left(\varphi_{p}-\varphi_{b}\right) / T_{i}$ increases with the electrostatic potential difference $e\left(\varphi_{i}-\varphi_{b}\right) / T_{i}$ being larger in the region of $\tau_{D} / \tau_{\text {transit }} \gtrsim 10^{2}$ of Figs. 3(a), 3(b), and 3(c).

\section{SUMMARY AND DISCUSSION}

In the Monte Carlo calculation the field ions and electrons are assumed to be Maxwellian distributions with temperature $T_{\text {field }}=100 \mathrm{eV}$. The axial densities of field ions and electrons are assumed to be uniform along the axis, i.e., $n_{\text {field }}=$ const. In order to obtain the simulation results with different parameters of $\tau_{D} / \tau_{\text {transit }}$, the density $n_{\text {field }}$ of field particles was changed. That is, the field temperature $T_{\text {field }}$ and test ion temperature $T_{i}$ input at $z=z_{i}$ were not changed throughout this article. The test ions input at $z_{i}$ move along the axis and receive Coulomb collisions with field particles and/or radial loss through the Monte Carlo method, which lead to the non-Maxwellian test ions in the mirror cell with different temperature form $T_{i}$ and nonuniform axial density profile as seen in Fig. 2. The electron distribution function in Eq. (1), was used to obtain the modified Boltzmann relation Eqs. (3) and (4) in the non-Maxwellian. Therefore, strictly speaking, the assumption that the field ions and electrons giving the Coulomb collisions with test ions are Maxwellian is not consistent. However, in the very collisional regime $\tau_{D} / \tau_{\text {transit }} \lesssim 1$, the distribution function of test ions is almost Maxwellian. On the other hand, in the collisionless regime $\tau_{D} / \tau_{\text {transit }} \gtrsim 10^{2}$ there is no population of test ions in the loss cone of $v_{\|}<0$. Therefore, the results of the Monte Carlo simulation in this article cover the various collisional regimes of the ion distribution function, i.e., from loss cone distribution function to Maxwellian distribution function. 
There is a potential maximum in the limit of weak collisions in Fig. 3, that is, the plug potential is highest in the limit of weak Coulomb collisions. The densities of ions and electrons in the central cell are desired to be high for a future nuclear fusion and the densities in the plug/barrier endmirror cells are required to be low for the high plug potential formation in a tandem mirror. The present tandem mirror experiments are operated under the balance between high plasma density in the central cell and low plasma density in the plug/barrier mirror cells.

In summary, we have shown that the saturated electrostatic potential is easily generated along a magnetic field line when the electron distribution deviates from Maxwellian and ions are accelerated in advance at $z_{b}$ in Fig. 1. The Coulomb collisions are required for the formation of a saturated electrostatic potential, that is, are required in order that a maximum point of the electrostatic potential exists in the region $z_{b}<z<z_{m}$ in Fig. $1 .{ }^{19}$ The results of this article have made clear that a saturated electrostatic potential can be created in a wide range of Coulomb collision frequencies. It is found that fewer Coulomb collisions make for a higher saturated electrostatic potential.

${ }^{1}$ G. I. Dimov, V. V. Zakaidakov, and M. E. Kishinevskij, Sov. J. Plasma Phys. 2, 326 (1976).

${ }^{2}$ T. K. Fowler and B. G. Logan, Comments Plasma Phys. Controlled Fusion 2, 167 (1977).
${ }^{3}$ D. E. Baldwin and B. G. Logan, Phys. Rev. Lett. 43, 1318 (1979).

${ }^{4}$ T. C. Simonen, S. L. Allen, T. A. Casper, J. F. Clauser, C. A. Clower et al., Phys. Rev. Lett. 50, 1668 (1983).

${ }^{5}$ D. P. Grubb, S. L. Allen, T. A. Casper, J. F. Clauser, F. H. Coensgen et al., Phys. Rev. Lett. 53, 783 (1984).

${ }^{6}$ T. Tamano, Phys. Plasmas 2, 2321 (1995).

${ }^{7}$ R. S. Post, K. Brau, J. Casey, X. Chen, J. Coleman, H. R. Garner, M. Gerver, S. Golovato, D. Goodman, W. Guss, S. Hiroe, S. Hokin, S. Horne, J. Irby, J. Kesner, B. Lane, T. Morgan, L. Pócs, E. Sevillano, D. Smatlak, D. K. Smith, J. Sullivan, R. P. Torti, and X. Z. Yao, in Plasma Physics and Controlled Nuclear Fusion Research 1986, in Proceedings of the 11th International Conference Kyoto, 1986 (International Atomic Energy Agency, Vienna, 1987), Vol. 2, p. 251.

${ }^{8}$ T. Kaneko, R. Hatakeyama, and N. Sato, Phys. Rev. Lett. 80, 2602 (1998).

${ }^{9}$ T. Kaneko, R. Hatakeyama, N. Sato, and S. Ishiguro, Phys. Plasmas 9, 1271 (2002)

${ }^{10}$ I. Katanuma, Y. Kiwamoto, M. Ichimura, T. Saito, and S. Miyoshi, Phys. Fluids B 2, 994 (1990).

${ }^{11}$ R. Minai, I. Katanuma, and T. Tamano, J. Phys. Soc. Jpn. 66, 2051 (1997).

${ }^{12}$ R. Minai, I. Katanuma, T. Tamano, and K. Yatsu, J. Phys. Soc. Jpn. 67, 876 (1998).

${ }^{13}$ I. Katanuma, Y. Tatematsu, K. Ishii, T. Saito, and K. Yatsu, J. Plasma Fusion Res. 77, 1085 (2002).

${ }^{14}$ I. Katanuma, Y. Tatematsu, K. Ishii, T. Saito, and K. Yatsu, Phys. Plasmas 9, 3449 (2002).

${ }^{15}$ K.-U. Riemann, J. Phys. D 24, 493 (1991).

${ }^{16}$ I. Katanuma, Y. Kiwamoto, Y. Tatematsu, K. Ishii, T. Saito, T. Tamano, and K. Yatsu, Phys. Plasmas 5, 1560 (1998).

${ }^{17}$ I. Katanuma, Y. Kiwamoto, K. Sawada, and S. Miyoshi, Phys. Fluids 30, 1142 (1987).

${ }^{18}$ G. A. Emmert, R. M. Wieland, A. T. Mense, and J. N. Davidson, Phys. Fluids 23, 803 (1980).

${ }^{19}$ I. Katanuma, Y. Kiwamoto, Y. Tatematsu, K. Ishii, T. Saito, K. Yatsu, and T. Tamano, Phys. Plasmas 4, 2532 (1997). 